\title{
Nut - Himmelsgöttin - Baumgöttin - Lebensgeberin
}

\author{
Von JAN BERGMAN
}

Verschiedene Umstände haben gemeinsam dazu beigetragen, dass der Ägyptologe sich in einer besonders günstigen Lage befindet, wenn es um das Studium einer reichen religiösen Symbolwelt innerhalb einer alten Hochkultur geht.

Erstens zeichnet sich die alte ägyptische Kultur schon von Anfang an dadurch aus, dass sie sich für das Wechselspiel von Bild und Schrift besonders interessiert hat. Davon zeugen sowohl der dauernde Bildcharakter der Hieroglyphenschrift wie auch das Zusammenspiel von Texten und Bildern in den Ritualszenen der Tempel sowie in den Totenbüchern und Jenseitsführern.

Zweitens konnte sich die mehr als 3000 Jahre dauernde altägyptische Kultur dank der geographischen Lage einer ungestörteren Entwicklung als die Nachbarkulturen des Nahen Orients erfreuen.

Drittens hat die für Ägypten eigene Konzentration auf das Jenseitsleben eine überaus reich entwickelte Vorstellungswelt mit Monumenten bleibender Art verbunden. Die Nekropolen waren ja als Wohnungen für die Ewigkeit gebaut.

Viertens haben die ungewöhnlich günstigen ökologischen Verhältnisse im Niltal dazu beigetragen, dass auch Dokumente und Gegenstände nicht so dauerhafter Art, vor allem Papyri und allerlei Grabausrüstungen, uns in erstaunlichem Umfang bewahrt sind.

Hier können wir natürlich nur einige kleine Einblicke in diese seltsam reiche und komplizierte ägyptische Symbolwelt geben. Beim Suchen einer für das Symposium geeigneten Symbolsphäre habe ich einige mit ,Leben“ zusammenhängende Vorstellungen ausgewählt. ,,Leben“ stellt ja nicht nur ein zentrales Thema in jeder Religion dar sondern kann gerade als Hauptthema der ägyptischen Religiosität gelten, die ihre Gedankenarbeit wie auch ihre materiellen Hilfsmittel und alle hochentwickelte Kunstfertigkeit dem Zusammenhang Leben-Tod-Leben gewidmet hat.

„Leben“ wird von Anfang an bis in die Spätzeit mit dem Anch-Zeichen ${ }^{1}$, das auch in frühchristlichem Zusammenhang als „Henkelkreuz" ${ }^{\text {"2 }}$ bekannt ist, wiedergegeben. Seine Ausgestaltung geben - mit bloss kleiner Varia-

1 Über Sinn und Gebrauch des Anch-Zeichens orientieren die Artikel ,Lebensschlei$\mathrm{fe}$ " in RÄRG $418 \mathrm{ff}$., "Anchzeichen" in Helck $1,268 \mathrm{ff}$. Cf. weiter die Sonderfor- schungen Fischer 1972, $5 \mathrm{ff} . ; 1973,16 \mathrm{ff}$; Baines $1 \mathrm{ff}$.

${ }^{2}$ Für Henkelkreuz-crux ansata, cf. Cramer, $1 \mathrm{ff}$., und Doresse $24 \mathrm{ff}$. 
tion - sowohl das protodynastiche Libationsgefäss wie auch die ptolemäische Opferplatte (als zentrales Zeichen) auf unserer Taf. 1 an. Wie dieses Zeichen ursprünglich zu erklären ist, bleibt umstritten. Wahrscheinlich stellt es einen Knoten oder eine Art Gürtel dar, wozu die sogenannte Isis-Schleife (oder Isis-Blut) ${ }^{3}$ eine ausführlichere Variante ausmacht.

Als Symbol eines gesteigerten Lebens, d. h. eines übermenschlichen, göttlichen Lebens, finden wir das Anch-Zeichen in verschiedenen Zusammenhängen und Verbindungen, natürlich auch als Amulette. Als ,Herren des Lebens" tragen die Götter das Anch-Zeichen in der Hand, was später auf die Könige übergegangen ist ${ }^{4}$. Aus der Namensgebung im Alten Reiche hat Junker ,,den Lebendigen" als gewöhnliches Götterepitheton belegen wollen ${ }^{5}$. Im wichtigen Spruch 80 der Sargtexte, wo eine imponierende Schu-Theologie entwickelt wird ${ }^{6}$, trägt Schu die Bezeichnung ,,derjenige, dessen Name Anch (Leben) ist". Dieser Gedanke wird - wie Winter trefflich ausgeführt hat ${ }^{7}$ - in den griechisch-römischen Tempeln breit weiterentwickelt, wobei die Verbindung von Anch-, Djed- und Uas-Zeichen als Bezeichnung des Opfers originell interpretiert wird. Als Trenner von Himmel und Erde hat Schu die Entstehung von Licht und Leben ermöglicht, und als Himmelsstütze ist er der Erhalter des Kosmos, der von Licht und Leben ernährt wird. Das Morgenritual der Tempel befestigt diese kosmische Ordnung, welche in fast unzähligen schönen Sonnenhymnen lebhaft beschrieben und gelobt wird. Die innige Verbindung von Licht und Leben lässt besonders die Sonnengötter als Lebensschöpfer und Lebensspender hervortreten. Wohl bekannt sind die zärtlichen Amarna-Szenen, in denen die Strahlen des Aton, in Hände mit dem Anch-Zeichen ausmündend, dem Pharao und seiner Königin das Licht-Leben überbringen.

Absichtlich soll in der Fortsetzung nicht das Anch-Zeichen als statisches Symbol für ,Leben“ - etwa ,,das ewige Leben“, das sich nicht verändert - in den Blickpunkt treten. Mehr kongenial mit der dynamischen Natur des sich bewegenden und verwandelnden Lebens dürfte ein Studium der wichtigsten Lebensprozesse sein. Das bedeutet, dass wir uns mit dem Vorstellungskomplex der Lebensgebung beschäftigen wollen, und zwar so, dass teils das Werden des Lebens, die Geburt des neuen Lebens und ihre Vorbereitung, teils das Meiden von Unterbrechungen dieses Lebens, d.h. die Überbringung von Lebensmitteln, unsere Aufmerksamkeit auf sich ziehen werden.

Wenden wir uns zuerst einem alten Kultgerät zu! Dem ganzen kultischen Handeln, das im alten Ägypten besonders reich entwickelt war und das wir in den späten Tempelanlagen in Details studieren können, könnte all-

${ }^{3}$ Cf. RÄRG s. v. Isisblut, 332 f.; Westendorf $144 \mathrm{ff}$.

${ }^{4}$ Cf. Fischer 1973, bes. $23 \mathrm{ff}$.

${ }^{5}$ Cf. Junker 1954, $32 \mathrm{f}$.
${ }^{6}$ Cf. de Buck 1 ff.; cf. auch Bergman 1970a, $54 \mathrm{ff},, 74 \mathrm{ff}$.

${ }^{7}$ Cf. Winter, bes. $76 \mathrm{ff}$. 
gemein als Ziel die Lebensgebung und Lebensbewahrung zugeschrieben werden. Ganz besonders gilt diese Charakterisierung dem funerären Kontext, wo das Leben als speziell bedroht aufgefasst wurde. Zum Grabkult gehört offenbar das protodynastische Libationsgefäss (cf. Taf. 1, Fig. 1 a-b), das vor 60 Jahren in einer thebanischen Nekropole gefunden wurde und sich jetzt im Metropolitan Museum befindet ${ }^{8}$. Hier hat man sowohl sinnvoll als auch handgreiflich - diese Eigenschaften sind für ein wirksames Kultsymbol nötig - die Vorstellung von Lebensgebung ausgestaltet. Dieses Kultgerät, das zum Begiessen mit Wasser bestimmt war - gleichgültig, ob es sich um Libation oder Reinigung handelte, - ist als Monogramm von zwei Hieroglyphen ausgeführt. Und zwar ist dies die Verbindung von AnchZeichen - hier in einer archaischen, detaillierteren Form dargestellt und von ,Ka-Zeichen" (was mit ,Geist, Persönlichkeit" aber auch mit „Nahrung" wiedergegeben werden kann). Hayes hat vorgeschlagen, hierin eine originelle Namensschreibung zu sehen, besonders da ein Beamter namens Anch-Kait(?) in der Regierung des Königs Den (1. Dynaste) belegt ist. Wenn auch die Möglichkeit einer solchen Deutung nicht zu verneinen ist, dürfte es dennoch klar sein, dass der primäre Sinn der besonderen Ausgestaltung des Gerätes gerade derjenige der Lebensgebung ist. In der vorliegenden künstlerisch ausgeformten Zusammenstellung der beiden Zeichen dienen die beiden Arme des Ka-Zeichens offenbar dazu, den Geber/Nehmer des Lebens anzugeben. Das Wasser, das in das Gefäss gegossen wurde, sollte das Gerät ausfüllen und somit die Szene der Lebensgebung lebendig machen, um danach durch den Griff des Anch-Zeichens auszulaufen und dem Toten/dem Kultdiener diese kultisch verwirklichte Lebensgebung zu vermitteln ${ }^{9}$. Dabei könnte natürlich auch das $\mathrm{Ka}$-Zeichen davon zeugen, dass diese Handlung besonders dem $\mathrm{Ka}$ des Verstorbenen/des Agierenden zugute kommen sollte.

Absichtlich habe ich in der gegebenen Interpretation die Frage des Agierenden offen gelassen. Im kultischen Interaktionsprozess sind nämlich Geber und Nehmer nicht so scharf von einander getrennt sondern - tiefer gesehen - durch ein und dieselbe wechselseitige Handlung mit einander verbunden. Wenn auch in unserem konkreten Fall Ka wohl in erster Stelle als Geber aufzufassen ist, kann er gut in einer späteren Phase - oder in einer früheren, als Bedingung der Lebensgebung - als Nehmer fungieren ${ }^{10}$.

\footnotetext{
8 Das Gerät ist z. B. Hayes 43 Fig. 31, abgebildet. $\mathrm{Cf}$. weiter die ausführliche Behandlung Fischer 1972, $5 \mathrm{ff}$.

9 Fischer, 1972, 34, vergleicht mit dem später belegten Verfahren bei den sogenannten Horusstelen, z. B. der Metternichstele, deren magische Sprüche vom übergegossenen Wasser aufgesogen wurden und so dank dem Wasser ihre gezielte Wirkungskraft erreichen sollten.
}

${ }^{10}$ Mit Hinweis auf einige protodynastische Amtssiegel, welche beim Titel, Einfanger des Ach" (zhri 3h) die Zeichen für Ach und $\mathrm{Ka}$ in ähnlicher Weise zusammenstellen wie die Zeichen für Anch und $\mathrm{Ka}$ auf unserem Gerät kombiniert sind (cf. Kaplony 3, Taf. 78 Nr. 296, Taf. 79 Nr. 299 usw.), könnte man auch hier die Deutung , Einfangen des Lebens" ( $z h n$ " $n h$ ) erwägen. 
In diesem Zusammenhang sind für die Deutungsmöglichkeiten zwei Umstände besonders zu beachten, teils die Richtung des Anch-Zeichens, teils die enge Beziehung, die in Ägypten zwischen Libation und Lustration vorliegt.

Man hat beobachtet, dass in den früheren Phasen der ägyptischen Kulturentwicklung beim Darreichen des Anch-Zeichens der Griff dem Nehmer zugewendet werden konnte, während in der klassischen Zeit die Götter zwar beim Tragen das Zeichen am Henkel halten, beim Darreichen aber mit wenigen Ausnahmen den Henkel der Nase des Königs nähern, so dass das Leben eingeatmet werden kann. Wenn nun beide Richtungen in der Frühzeit möglich waren, ist somit eine doppelte Deutung bei unserem Gefäss möglich ${ }^{11}$.

Auch die enge Beziehung zwischen Libation und Lustration ${ }^{12}$, von der später Texte und Darstellungen deutlich zeugen, dürfte eine solche doppelte Interpretationsmöglichkeit nahelegen. Bei der Reinigung besprengt man sich oft selbst, wenn auch z. B. die Szenen, welche die sogenannte ,Taufe des Pharao" darstellen, Götter als Reiniger-Beleber des Pharao durch einen Strom von Anch- und Uas-Zeichen auftreten lassen. In ähnlicher Weise dürfte der älteste Sohn/der Ka-Priester im funerären Kult agiert haben. Aus den vielen Reinigungstexten, die von der tiefgehenden Wirkung des mit göttlichen Kräften gefüllten Wassers reden, wählen wir folgenden aus: ,,Ich reinige dich mit diesem Wasser allen Lebens und Heils, aller Gesundheit und aller Freude" (Naville Taf. 63). Ganz in Übereinstimmung mit dem Wortlaut dieses Textes wird dieses wirksame Wasser in den Reinigungsszenen geläufig gerade mit Strahlen von wechselnden Anch- und UasZeichen - etwa als ,,heiliges/machtvolles Lebenswasser“ - wiedergegeben.

Mit diesen Andeutungen müssen wir uns hier begnügen und zusammenfassend feststellen, dass unser Anch-Gefäss als evidentes Symbol der Lebensgebung dasteht, wobei vermutlich $\mathrm{Ka}$ besonders betont wird. Wer aber als ursprünglicher Geber des Lebens gilt, sagt dieses Gefäss nicht aus. In einer Gruppe von spätzeitlichen Opferplatten aus Achmim (cf. Taf. 1 Fig. 2) - etwa 3000 Jahre später als unser Gefäss! - können wir dank der Seitenszenen eine Antwort finden. Zu dem mit diesen Darstellungen verbundenen Thema kommen wir aber unten zurück. Wir wollen hier nur feststellen, dass das hier erscheinende zum Libationsgefäss verwandelte Lebenszeichen, aus dessen Henkel Wasser gegossen wird, mehrmals schon im Neuen Reiche zu belegen is $t^{13}$. Anch kann vereinzelt auch personifiziert auftreten, wobei seine Hände Libationsgefässe halten ${ }^{14}$.

$11 \mathrm{Zu}$ bemerken ist, dass beim Schreiben des Namens Anch-kai(?) in den Amtssiegeln (cf. Kaplony 3, Taf. $73 \mathrm{Nr} .276 \mathrm{~A}$, Taf. $74 \mathrm{Nr}$. 276B, Taf. 79 Nr. 298) das Anch-Zeichen in der entgegengesetzten Richtung dargestellt ist. Für Belege der beiden Richtungen beim Darreichen des Anch-Zeichens cf. Brunner 71.

12 Cf. RÄRG s. v. Reinigung, $633 \mathrm{ff}$.

13 Cf. Jéquier 1922, $137 \mathrm{ff}$.

14 Cf. Fischer 1972, 35 Fig. 9. 
Es gibt eine ägyptische Gottheit, die in unserem Zusammenhang von besonderem Interesse ist, und zwar - wie der Titel sagt - die Göttin Nut. Wenn Schu oben als "derjenige, dessen Name Anch (Leben) ist" vorgestellt wurde, lässt sich diese Tatsache gut mit Nut als der Lebensgeberin par excellence vereinen. Die Trennung von Himmel und Erde, die als die hauptsächliche Leistung des Gottes Schu gilt, wird geläufig mythologisch ausgelegt: es handelt sich um den Sohn Schu, der die in Coitus vereinigten Eltern Nut und Geb auseinanderbringt und so als "Leben“ ins Licht hervortritt. Als Mutter des „Lebens“ steht so Nut als Lebensgeberin da. Und der Sohn Schu - ob als Lebenslicht oder als Lebenshauch verstanden - ist enger mit seiner Mutter-Himmel als mit seinem VaterErde verbunden.

Ehe wir auf zwei Vorstellungskomplexe näher èingehen, die Nut mit den beiden wichtigen Lebensprozessen, die oben angegeben worden sind, eng verbinden, ist eine kurze Darstellung dieser Göttin am Platz, wobei solche Züge ihres Wesens besonders beachtet werden sollen, die mit den folgenden Ausführungen zusammengehören können.

Nut ist vorzüglich als Himmelsgöttin bekannt. Als solche hat sie ,die Erde und alle Dinge in ihren Armen beschlossen", sagt ein Pyramidentext (Pyr. 782 d), wobei hinzugefügt wird, dass sie ,,den König als einen unvergänglichen Stern, der in ihr ist, gesetzt hat" (782 e). Eine alte Benennung der Nut ḩ $3 b 3 s$ wird demnach als ,Eine mit Tausend Seelen“ gedeutet, da die Seelen der Toten als Sterne am Himmel weiterlebend vorgestellt wur$\operatorname{den}^{15}$. Wie die Sterne dorthin gelangen, sagt ein später Text: ,Sie gehen in den Mund (scil. der Himmelsgöttin) ein und kommen wieder aus ihrer Scheide hervor" ${ }^{\prime 16}$. Nach Amduat erscheint die Sonne am Morgen ,,zwischen den Schenkeln der Nut" ${ }^{17}$. Nut trägt als ihre gewöhnlichste Bezeichnung ,Nut, die Grosse, welche die Götter gebiert“ oder ,Nut, die Mutter der Götter". Eine Sondervorstellung, die ihre Fruchtbarkeit hervorhebt und die seit dem Neuen Reiche zu belegen ist, lässt Nut als ,Mutterschwein, das seine Ferkel gebiert und wieder verschlingt" erscheinen ${ }^{18}$. Bekannt ist das Nutbild im Kenotaph Sethos' I. in Abydos ${ }^{19}$, das ausdrücklich diese Funktionen der Göttin darstellt und beschreibt. Auch am Ende des Amduat liegt ein Himmelsbuch vor, das , Nut-buch“" genannt werden kann und das auch an der Decke der Sargkammer Ramses' IV. zu finden ist ${ }^{20}$. Der Umstand, dass Nut sowohl als Taghimmel als auch als Nachthimmel betrachtet wird, gibt ihr eine doppelte Reihe von Schwangerschafts- und Geburtsrollen. Diese Feststellung kann einen geeigneten Hintergrund für die Behandlung des ersten Vorstellungskomplexes der Lebensgebung ausmachen.

${ }_{15}$ Cf. Bergman 1970, 31 Anm. 2; 35 Anm. 1.

${ }_{16}$ Mariette Taf. 46.

17 Jéquier 1894, 136; cf. Hornung 3,24 Z. 278.
${ }^{18}$ Cf. Grapow 45 ff.; Bergman 1974, 91 ff.

19 Cf. Frankfort Taf. 81.

${ }^{20}$ Hornung $1963 \mathrm{f}$., $194 \mathrm{f}$. 


\section{Nut, die Lebensgeberin, als Himmelsgöttin und Mutter des Toten}

Die kurze Übersicht hat uns die Himmelsgöttin Nut als Gebärerin der Sonne vorgestellt. Dies bedeutet, dass sie neben der kosmogonischen Beziehung auf Schu vor allem als Mutter des Re dasteht. Dass diese Rolle für den Totenkult äusserst wichtig war, da der Gestorbene wünschte, ,,am Tage hervorzugehen wie $\mathrm{Re}^{\mathrm{s}}$, ist selbstverständlich. Der Wiedergeburt Res sollte der Tote dank allerlei funerären Ritualen teilhaftig sein. Das erste Ziel dieser umfassenden Zeremonien war jedoch die Osiriswerdung. Als Osiris NN sollte der Tote vor Osiris als schon Gerechtfertigter beim Totengericht in der Unterwelt vorgestellt werden. Auch für die Verwirklichung dieser Rolle war Nut für den Toten die wichtigste Gegenspielerin. Als ,Mutter der Götter ${ }^{66}$ war sie nämlich nach der erweiterten heliopolitanischen Göttergenealogie, der klassischen Götterenneade, die Mutter des Osiris, wie auch die des Seth, der Isis und der Nephthys. Sowohl für die Teilnahme am Jenseitsleben, wo Osiris die Zentralgestalt war, wie auch für die Beteiligung am Diesseitsleben, dessen Hauptvertreter Re war, machte für den Toten die innigste Beziehung zu Nut, als Vereinigung mit ihr und Geburt aus ihr verstanden, eine notwendige Bedingung aus.

Dieser Vorstellungskomplex hat in mehreren Hinsichten den Totenkult geprägt, wie vor allem Rusch aufgezeigt hat ${ }^{21}$. Hören wir die Zeugnisse einiger Texte! ,Nut, breite dich über deinen Sohn. Schütze ihn vor Seth. Schirme ihn, Nut. Du bist gekommen, dass du deinen Sohn schützest. Du bist gekommen, damit du diesen Grossen schirmst“ (Pyr. 777). ,Deine Mutter Nut hat sich über dich gebreitet. Sie schützt dich vor allen schlechten Dingen. Sie schirmt dich vor allem Übel, denn du bist der Grösste unter ihren Kindern" (Pyr. 825). Diese Aussagen, die in mehreren Varianten schon in der ältesten Totentextsammlung, d. h. in den Pyramidentexten, vorliegen, behandeln nicht ausdrücklich die Geburt sondern stellen primär die Göttin als Schützerin und Schirmerin des Toten und ais Garantin für sein Leben im allgemeinen dar. „Nut, du warst ein Ach, du warst eine Macht schon im Leibe deiner Mutter Tefnut, bevor du geboren warst. Schirme diesen NN, damit er nicht stirbt" (Pyr. 779). Dieser Text verankert die Leben schützende Funktion tief im Wesen der Göttin. Nut ist selbst schon pränatal, d.h. ursprünglich, ihrer tiefsten Natur nach, ,der grosse Ach“ (vgl. Pyr. 1), wobei Ach, der höchste Rang unter den Lebenden in der Unterwelt, sehr eng mit dem Leben in höchster Potenz verbunden ist. Hier wäre es verlockend, eine Nut-Ach-Achet-Theologie - Achet ,Horizont" kann als feminine Form des Ach verstanden werden - zu entwickeln, von der m. E. mehrere funeräre Texte zeugen. Wir müssen uns jedoch hier damit begnügen, diesen Zusammenhang nur angedeutet zu haben ${ }^{22} \mathrm{Zu}$

21 Rusch $1 \mathrm{ff}$.

${ }_{22}$ Cf. Englund 1978, bes. $30 \mathrm{f}$, 40. Da E. in diesem Teil ihrer Achuntersuchungen, deren
Fortsetzung vorbereitet wird, 3 ht-,,Horizont" nicht näher behandelt, tritt diese NutAch-Achet-Theologie, die sich indessen mit 
bemerken ist weiter, dass der zitierte Passus nur die erste Strophe einer längeren Textpartie ausmacht, in der die zweite Strophe diese Natur der Göttin mit ihrem Namen verbindet und die dritte - und letzte - in folgendes Gebet ausmündet: ,Verkläre diesen $N \mathbb{N}$ in dir, damit er nicht stirbt!'23 Diese Verklärung, die wortgetreu mit , Ach-Machung" wiederzugeben ist, gibt den Tiefsinn der besonderen Lebensgebung der Göttin Nut an. Es geht nicht nur um einen Wiedergewinn des gewöhnlichen Lebens sondern um die Gewinnung des neuen gesteigerten Lebens eines Ach, das Nut als , die grosse $\mathbf{A c h}{ }^{6}$ am sichersten schenken kann.

Wenn auch die Geburt in den zitierten Texten nicht direkt hervorgehoben wird, handelt es sich jedoch offenbar tiefer gesehen um die Geburt aus Nut. Die Verklärung soll in Nut, d. h. in ihrem Leib, verwirklicht werden, und der Tote wird geläufig als ihr Sohn bezeichnet, ja, er tritt als ihx Lieblingssohn hervor. Auf dem Sarge des Teti wird sogar versichert, dass Nut den Pharao mehr als ihre eigene Mutter Tefnut liebt (Pyr. 5). Einige Texte deuten ausdrücklich diese Geburt des Pharao als Verwandiung zu einem Sterngott, der am Himmel hervortritt. Interessant ist, dass diese Stellung als ,am Leibe der Himmelsgöttin Nut" oder, , in ihrem Leibe“" bestimmt wird ${ }^{24}$. Bei der Redaktion der Pyramidentexte fand aber eine Osirisierung statt, so dass die Osiris-Rolle des Pharao bei dieser Sohnschaft $\mathrm{zu}$ Nut hier vorherrschend ist. Typisch ist folgende Aussage: , Nut gibt mir ihre Arme wie das, was sie für Osiris getan hat" (Pyr. 1090). Zur selben Zeit kann aber auch seine Re-Rolle betont werden, wenn Nut als Taghimmel gedeutet wird. Ein Sargtext stellt fest: ,Nut hat dich geboren gemäss der Geburt des $\operatorname{Re}^{\text {if }}$ (CT III, 398), und derselbe Gedanke wird schon in den Pyramidentexten belegt (Pyr. 1688, 1835). Den Zusammenhang der beiden Vorstellungen gibt folgender Passus an: ,Dieser $N N$ liegt da, indem er jeden Tag geboren und empfangen wird; er ist gestern weggegangen, er ist heute wieder gekommen" (CT IV, 88-89; cf. auch Pyr. 1703 ff.).

Dieser Vorstellungskomplex hat nun seinen konkreten Audruck in der Grabkammer gefunden. Die Placierung von Texten wie: ,Ich (scil. Nut) breite mich über dich aus" unter dem Dach charakterisiert deutlich die Decke des Grabes selbst als Himmel. Aber auch an den Wänden des Grabes treten Texte auf, die davon reden, dass Nut den Toten umschliesst, vereinigt, schützt usw. In diesem Fall gilt offenbar das ganze Grab als der Himmel/Nut. Somit befindet sich der Tote sowohl under dem Himmel/ Nut - oder am Himmel/Nut - wie auch im Himmel/Nut. Nut hat sich als Grabdecke über den Toten gelegt. Hier tritt eine wichtige MikrokosmosVorstellung an den Tag. Eine solche liegt aber auch im Falle des Sarges vor. Der Pyramidentext 616 kombiniert beide und stellt mit einer drei-

ihren bisher gewonnenen Resultaten (205ff.) sehr gut verbinden lässt, nicht besonders hervor.
${ }^{23} \mathrm{Cf}$. Rusch $4 \mathrm{f}$.

24 Cf. ib., 15 ff. 
fältigen Namensspielerei - es werden zwei verschiedene Wörter für „,Grab" gebraucht - fest: ,Du wirst gegeben deiner Mutter Nut in ihrem Namen 'Grab', sie umschliesst dich in ihrem 'Sarg'; du kommst zu ihr (zum Himmel) hinauf in ihrem Namen 'Grab'." Diese konkreten Anschauungen können sowohl die grosse Frequenz der Nut-Texte wie ihre bewussten Placierungen erklären. So wird auch die sonst merkwürdige Tatsache verständlich, dass Nut in diesen Texten eine weit bedeutendere Rolle als Geb spielt, der jedoch als Unterweltrichter von grosser Wichtigkeit sein soll.

Mit dem Auftauchen einer reichen Bildausschmückung der Särge im Neuen Reiche eröffnen sich neue Möglichkeiten, diese Vorstellungen auch bildmässig zu belegen. So geben die variierenden Deckendekorationen der Gräber deutlich an, dass die Decke als Himmel anzusehen ist, wenn auch die Göttin Nut selbst hier nicht so oft in anthropomorpher Gestalt auftritt. (Die interessante Nut-Darstellung an der Decke des thebanischen Grabes 216 wird unten in einem anderen Kontext behandelt.) Mehrere Königsgräber zeigen aber stattliche Nut-Darstellungen auf, z. B. die Decke der Sargkammer Ramses' IV. Die Särge sind jedoch weit ergiebiger. Hier findet sich Nut in vielen Rollen. Als Schirmerin des Toten wird sie geläufig mit weit ausgebreiteten Flügeln zentral oben am Sarg dargestellt. Die gewöhnliche kosmische Szene, in der Nut, soeben von ihrem Gatten/ Begatter Geb getrennt, sich über ihn nackt ausbreitet, findet sich oft als Seitenszene in der Herzgegend placiert. Diese wichtige Darstellung ${ }^{25}$ fixiert den kosmisch-mythischen Kontext der Wiedergeburt des Toten, an den die anderen Nut-Bilder anspielen.

Besonders wichtig sind die grossen Darstellungen der Göttin Nut auf der Innenseite des Sargdeckels. Diese zeugen, auch von den Texten gestützt, davon, dass auch der Sargdeckel selbst, nicht nur der ganze Sarg, mit der Himmelsgöttin identifiziert wird. Wie der Deckel breitet sich Nut, etwa in derselben Grösse wie der Tote, über diesen aus. Unter mehreren Belegen ${ }^{26}$ wählen wir zwei aus, die sich auf unserer Taf. 2 wiederfinden. Figur 1, die summarische Abzeichnung eines schön ausgeschmückten Leidener

25 Bergman, 1975, gibt einige Hinweise auf
solche Darstellungen (12 ff.) und hebt ihre
Verbindung mit Empfängnis und Geburt
hervor.
26 Im typologischen Atlas der Särge, Mu-
mienkisten (usw.) von Schmidt finden wir
folgende Belege, die sich leicht erweitem las-
sen (die beiden Nummern beziehen sich auf
Seite bzw. Abbildung.): 169(930), 173(958),
206(1177), 218(1238), 226(1302), 229(1321),
230(1330), 232(1340), 233 (1344) und - je-
doch mit der Göttin im Profil - 168(923,
924), 186(1032). Auch ihre Darstellung auf
dem Sargboden ist für uns wichtig. Wenn Nut nicht nur mit dem Deckel sondern mit dem ganzen Sarg identifiziert wird als ,,diejenige, die den Toten umschliesst" (usw.), ist ihre Placierung auch auf dem Sargboden sinnreich. Hier sind $199(1125)$ und 231 (1329) wegen der Frontalität besonders zu bemerken. Cf. weiter $140(718), 177(983), 183(1014)$, 192 (1082), $206(1178), 218(1239)$. In diesem Fall wird Nut bisweilen von anderen Göttinnen, und zwar besonders von Amentet, der Göttin des Westens, ersetzt. Amentet dürfte hier beim Empfangen des Toten im Westen etwa als eine Sondererscheinung der Nut gelten. 
Sarges, zeigt die sich über den Toten ausbreitende Himmelsgöttin en face und nackt. Diese beiden Darstellungsweisen, Frontalität und Nacktheit, die in der ägyptischen Kunst überhaupt selten sind, dienen dazu, den Zusammenhang mit Empfängnis und Geburt hervorzuheben ${ }^{27}$. Wie oben beobachtet wurde, charakterisiert die Nacktheit der Göttin gerade die kosmische Nut-Geb-Szene. Die Placierung der beiden Sonnen am Leibe der Nut deuten Empfängnis bzw. Geburt an. Zu beiden Seiten der Göttin sind die je 12 Stundengöttinnen der Nacht und des Tages dargestellt, was die kosmische Dimension noch mehr betont. Durch die Vereinigung mit der Göttin und die Geburt aus ihr sollte der Tote sicher sein, des Sonnenlaufes sowohl der Nacht als auch des Tages teilhaftig zu werden.

Figur 2 (Taf. 2) zeigt Nut dagegen bekleidet und im Profil. Die Placierung der Darstellung ist jedoch dieselbe wie in Figur 1. Die Haltung der Arme deutet eine Umarmung des Toten an. Besonders wichtig für uns ist aber die ganz merkwürdige Ausgestaltung ihres Kleides. Hier erkennen wir leicht die sogenannte Isis-Schleife, die hier wohl als eine umständlichere und dekorativere Form des Anch-Zeichens aufzufassen ist. Somit tritt hier die Himmelsgöttin Nut als das Leben selbst hervor! Eine solche Darstellungsweise ist natürlich eine ausserordentliche Stütze für die Behauptung, dass Nut auch die Lebensgeberin par excellence ist.

Ehe wir diesen Abschnitt über Nut, die Lebensgeberin, als Himmelsgöttin und Mutter des Toten abschliessen, um zum zweiten angekündigten Motiv überzugehen, sei auf den Tatbestand hingewiesen, dass Nut auch am Boden des Sarges, meistens als Gegenhimmel gedeutet, auftritt. So umschliesst sie auch auf diese Weise den Toten, der sich in ihr befindet.

\section{Nut, die Lebensgeberin, als Baumgöttin und Ernährerin des Toten}

Das hier zu behandelnde Motiv findet sich auch nicht selten auf Särgen dargestellt, lässt sich aber ausserdem in verschiedenen anderen Zusammenhängen belegen, was von der Popularität dieser Darstellung und der dahinterstehenden Vorstellung gut zeugt. Sehr gewöhnlich ist die betreffende Szene in Vignetten zu den Kap. 57 und 59 des Totenbuchs, aber auch auf Grabstelen, in Grabreliefs, auf Opferplatten usw. ist das Motiv oft zu finden. Während der schon behandelte Typus von Lebensgebung sozusagen ex ovo beginnt und das Werden des Lebens in den Blickpunkt stellt, betont der zweite Typus die Fortdauer des aus Nut geborenen Lebens. Als Geberin des Lebens ist Nut auch für dessen Fortsetzung verantwortlich. Das sich von der Empfängnis bis auf die Geburt organisch entwickelnde Leben muss auch nach der Geburt weiter wachsen. Dafür sind Lebensmittel nötig. Solche bringt Nut/die Sykomore. Sie hält das Leben am Leben.

Wenn ein Pyramidentext (Pyr. 718) sagt: ,,Der König lebt vom Baum mit süssen Früchten", ist es unsicher, ob hier die Sykomore gemeint wird.

${ }^{27}$ Cf. Bergman 1974, bes. $100 \mathrm{ff}$. 
Vielleicht geht es eher um die Dattelpalme. Die Sykomore wird ansdrücklich in ein paar anderen Texten erwähnt, wobei indessen andere Funktionen als das Ernähren von Bedeutung sind: sie dient als Schutz und Ruheplatz sogar für die Götter (Pyr. 1485) oder wird als ein merkwürdiger uralter Baum am Osthimmel, auf dem die Götter sitzen, beschrieben ${ }^{28}$.

Erst mit dem Neuen Reiche lassen sich bildliche Darstellungen von der Lebensgeberin Nut/Sykomore belegen, wie es auch mit dem früher behandelten Motiv der Fall war. Auf unserer Taf. 3 finden sich vier Varianten dieses Motivs. Aus Figur 1, einer spätzeitlichen Reliefdarstellung in Äthiopien, wo bloss zwei Wasserstrahlen aus dem Baum hervorquellen, kann man keine Aussage über den Geber/Geberin ziehen. Figur 2 dagegen zeigt deutlich durch die aus dem Baum halbwegs hervorwachsende Göttinnengestalt, dass eine Göttin als Geberin von Getränk und Nahrung auftritt, und der die Lebensmittel emprangende $\mathrm{Ba}$ markiert, dass es um einen funerären Kontext geht. Die Göttin bleibt jedoch hier anonym. Die Göttin in Figur 4 wird eindeutig als Nut gekennzeichnet. Da sie aber hier ganz freistehend vom Baum dargestellt wird, kann man ohne Parallelmaterial nicht auf eine Identität zwischen Göttin und Baum schliessen. Figur 3 ihrerseits zeugt gut von einer solchen Identität, bezeichnet aber die Göttin als Amentet, d. h. als Göttin des Westens. Dank einer fast überwältigenden Menge von Parallelen, deren Mehrzahl der Baumgöttin - aus praktischem Grunde gebrauche ich diese oberflächliche Bezeichnung - den Namen Nut zuschreiben, ist aber ihre Identität klar.

Von grösster Bedeutung für die weite Verbreitung des Motivs und seine feste Verbindung mit Nut dürte seine Beliebtheit in den Vignetten und den diese begleitenden Texten einiger Kapitel des Totenbuches, vor allem der Kap. 57 und 59, gewesen sein. Im letztgenannten Kapitel lesen wir: ,Heil dir, Sykomore der Nut! Gib Wasser und Luft, die in dir sind!“ Und auf der Grabstele des Ineni (Urk. IV,65) beschreibt der Tote sein Schicksal nach dem Tode so: ,,Meine Seele [. . . ] durchschreitet ihren Garten nach ihrem Belieben. Ich verwandle mich. Ich komme hervor zum Tage. Ich erfrische mich unter meinen Sykomoren, ich emähre mich von dem, was Nut schafft. Ich trinke Wasser nach meinem Belieben." ${ }^{29}$ Die Vignetten zeigen die Baumgöttin Nut, die dem Toten/seinem Ba Wasser aus zwei Libationsgefässen spendet. Bisweilen wird das eine Gefäss von einer Opferplatte mit allerlei festen Nahrungsmitteln ersetzt, die eine aus dem Baum hervortretende Hand darbringt.

$\mathrm{Zu}$ bemerken ist der Tatbestand, dass die roten Früchte der Sykomore, welche die natürlichste Gabe der Göttin sein sollten, nur selten dem Toten

${ }^{28}$ Für diese, ,seitlich verbrannte, innen verkohlte, die richtig ausgeblutete" Sykomore, cf. Moftah, $40 \mathrm{ff}$, der hierin die ehrwürdige Hathor-Sykomore südlich von Memphis erkennen will.
29 Wallert 86; der Passus zeigt eine Lucke, die hier nach Paralleltexten rekonstruiert ist. 
dargeboten werden. Das Wasser spielt hier die Hauptrolle, was sowohl die Vignetten als auch die Texte betonen. Dies hängt offenbar von der konkreten Lage der Nekropole ab. Die Grabanlagen waren ja am Wüstenrand gelegen, wo der Durst sehr drückend war. Vereinzelte Bäume dienten durch ihre Wurzelnetze als Wassersammler ${ }^{30}$. Baum and Teich werden geläufig in den Grabanlagen zusammengestellt. Ob wirklich im Neuen Reiche Bäume in den thebanischen Nekropolen in solcher Menge vorhanden waren, wie es die Grabreliefs usw. darstellen, ist umstritten. Vielleicht bilden die Szenen einen Idealzustand $a b$, welcher nur in den Gärten auf der Ostseite des Nils zu dieser Zeit verwirklicht werden konnte und - was die Nekropolen anbelangt - der Frühzeit und dem mythischen Vorbild (vgl. die Rolle des Baumes am Osirisgrab in Abydos usw.) angehörte. Es ist somit nur natürlich, dass das Wasser die Hauptsache war, was übrigens auch für die Spätzeit das formelartige Gebet an Osiris auf den Grabsteinen bezeugt, dass er dem Toten kühles Wasser bringen solle ${ }^{31}$.

Neben dem Wasser spielt auch die Luft eine wichtige Rolle in diesem Kontext. ,,Gib Wasser und Luft, die in dir sind" lautet das schon zitierte Gebet. Vielleicht steckt dahinter der Gedanke, dass das Zweigwerk die Luft aufsammelte und dem Toten vermittelte wie es das Wurzelnetz mit dem Wasser tat. Bezeichnend ist folgender Wortlaut in einem späten Osirisritual: ,,Seine Mutter Nut ist die Sykomore, die ihn schützt und seinen Ba in ihren Zweigen verjüngt." ${ }^{32}$ Die Vorstellung von der Verjüngung des Ba gerade in den Zweigen könnte m. E. eine solche Deutung stützen. Auch im. späten Choiakritual hat die hier behandelte Vorstellung einen Niederschlag gefunden, indem Osiris gerade auf Sykomorenzweigen gebettet werden soll. Bisweilen hält der Tote in den Vignetten die Hieroglyphe des Windes in der Hand, was ihn als Empfänger des Lufthauches auszeichnen soll. Hier wäre vielleicht an die im Neuen Reiche veränderte Richtung beim Darreichen des Anch-Zeichens zu erinnern. Beim protodynastischen Libationsgefäss dient der Griff des Zeichens funktionell als Ausgiesser bei der Wasserspende. Wenn es aber um die Luft geht, könnte beim Darbieten des Anch-Zeichens/des Lebenshauches die Luft durch das Loch des Henkels zur Nase gelangen.

Aus der ptolemäischen Zeit stammt eine Gruppe von Opferplatten, die fast ausnahmslos in der Gegend von Achmim zuhause sind ${ }^{33}$. Schon der Umstand, dass die darauf aufgenommenen Texte gewöhnilch die oben

30 Cf. Wallert $97 \mathrm{f}$.

${ }^{31} \mathrm{Cf}$. Parrot $126 \mathrm{ff}$. In diesem Buch, das den Hintergrund des ,,refrigerium " in der Liturgia Gallicana zeichnen will, spielt unsere ägyptische Tradition eine hervorragende Rolle (84 ff.).

${ }^{32}$ Cf. Junker 1913, 51. Für die besonderen Baumtraditionen, die mit dem Osirisgrab auf dem Abaton verbunden waren - und die auch bei Plutarch erwähnt werden - sei auf die weiteren Ausführungen Junkers, $51 \mathrm{ff}$., hingewiesen.

${ }^{33}$ Cf. Kamal Nr. 23.160-23.172, Taf. 41-44. Ein vergleichendes Detailstudium dieser symbolreichen Opferplatten könnte weitere kleine Beiträge zu unserer Lebensgebungsymbolik geben. 
zitierte Totenbuchstelle (Kap. 59; inzwischen auch ähnliche Stellen aus Kap. 60 und 62) anführen, macht diese Gruppe für uns interessant. Ausserdem zeigen mehrere unter ihnen einen lockenden Symbolreichtum auf. An einem ausgewählten Beispiel (Taf. 1 Fig. 2) wollen wir die Verwendung und die tiefere Funktion eines solchen Kultgeräts beleuchten. Typisch für die Kontinuität der ägyptischen Vorstellungswelt und Kultpraxis ist die Tatsache, dass manches hier an das protodynastische Libationsgefäss erinnert, das wir anfangs behandelten. Absichtlich haben wir auch diese beiden Kultgeräte, die von einander etwa 3000 Jahre entfernt sind, auf dieselbe Tafel placiert.

Diese steinerne Opferplatte behält die ursprüngliche Form der geflochtenen Matte, auf welche man die Opfer legte ${ }^{34}$. Diese Form ist übrigens mit der Hieroglyphe für ,Opfer" ( $h t p$ ) identisch. Es handelt sich um ein Gefäss, in welches Wasser über die dargelegten - oder vielleicht nur abgebildeten - Opfergaben ausgegossen wurde, und zwar so - was wohl in unserem Fall das Wichtigste war - dass die dort angebrachten Texte und Bilder vom Wasser belebt wurden. Durch einen Ausguss, gebildet aus dem brotförmigen Aufsatz des htp-Zeichens, kam so dieses lebensvolle Wasser dem Toten zugute. Dass dieses Libationsverfahren das Hauptgewicht trägt, beleuchtet gut die Ausschmückung. In der Mitte finden sich drei Libationsgefässe und dazwischen allerlei Opfergaben. Von diesen drei Gefässen zeigt das mittlere eine Sondergestalt auf. Hier erkennen wir die oben kurz erwähnte Anchi-Vase, die einfach wie ein Anch-Zeichen aussieht, das mit einer Mündung, identisch mit derjenigen der beiden anderen Gefässe, versehen ist. Diese Anchi-Vase ist nun auf unserer Opferplatte an einem Teich ,gepflanzt", so dass das Wasser des Teiches - mythisch als das Urwasser Nun, als der Nil und als der Ausfluss des Osiris gedeutet - von der Anchi-Vase ,aufgesogen“ dem Toten zugute kommen kann. Bewusst habe ich bei der Beschreibung die beiden Verba ,,pflanzen" und ,,aufsaugen" für die Aktivitäten der Anchi-Vase gebraucht. Die beiden uns bekannten Seitenszenen - es geht um die Göttin Nut, die als Sykomore dem Toten als Lebensgeberin-Ernährerin dient - stellen ja unzweideutig auch die Zentralszene in diesen Zusammenhang hinein. Somit ist man berechtigt, auch diese Anchi-Vase als eine konkrete Erscheinungsform der Wasser spendenden Göttin Nut zu verstehen. Wie oben angedeutet wurde, stellen auch die Texte, die auf dieser Platte stehen - es handelt sich um Auszüge aus dem Kap. 59 (rechts) und dem Kap. 62 (links) - die Identifikation der Göttin/des Baumes als Nut sicher.

Merkwürdig ist nun, dass Nut/die Sykomore in der rechten Szene ganz anthropomorph, in der linken aber hauptsächlich dendromorph dargestellt wird - und dies ist fast ausnahmslos für die ganze Gruppe der Opfer-

${ }^{34}$ Für die Entwicklung der Opferplatten cf. RÄRG s. v. Opferplatte $557 \mathrm{ff}$. 




Fig, l:a

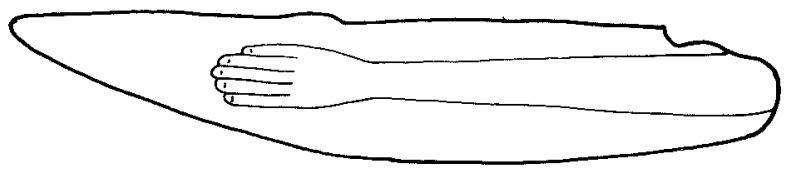

Fig. 1:b

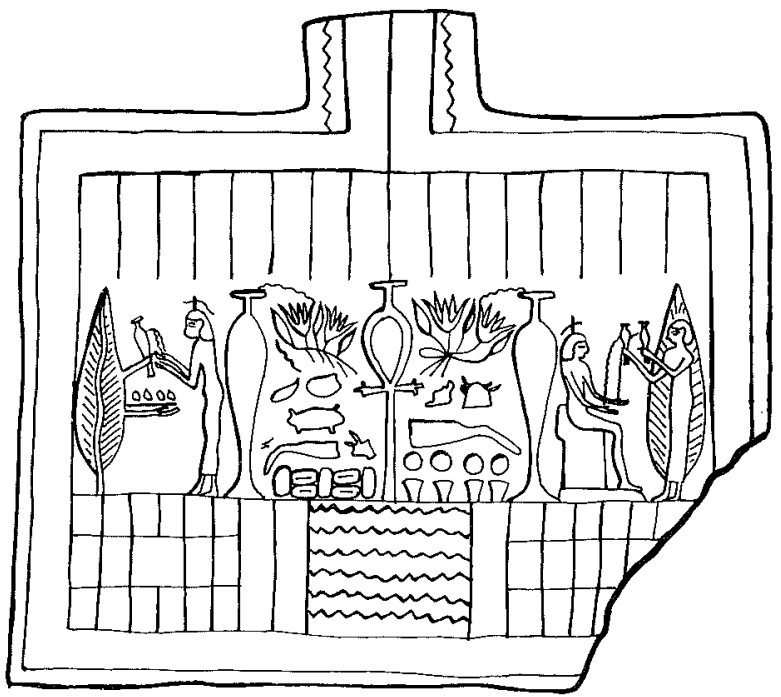

Fig. 2

Taf. 1. Kultgeräte.

Fig. $1 \mathrm{a}-\mathrm{b}$. Protodynastisches Libationsgefäss aus der thebanischen Nekropole (Metropolitan Museum 19.2.16). Cf. Fischer 1972, $5 \mathrm{ff}$.

Fig. 2. Opferplatte aus Achmim Nr. 23.162 im Kairoer Museum (ptolemäisch). Cf. Kamal 1, $120 \mathrm{f}, 2$, Taf. 41. 


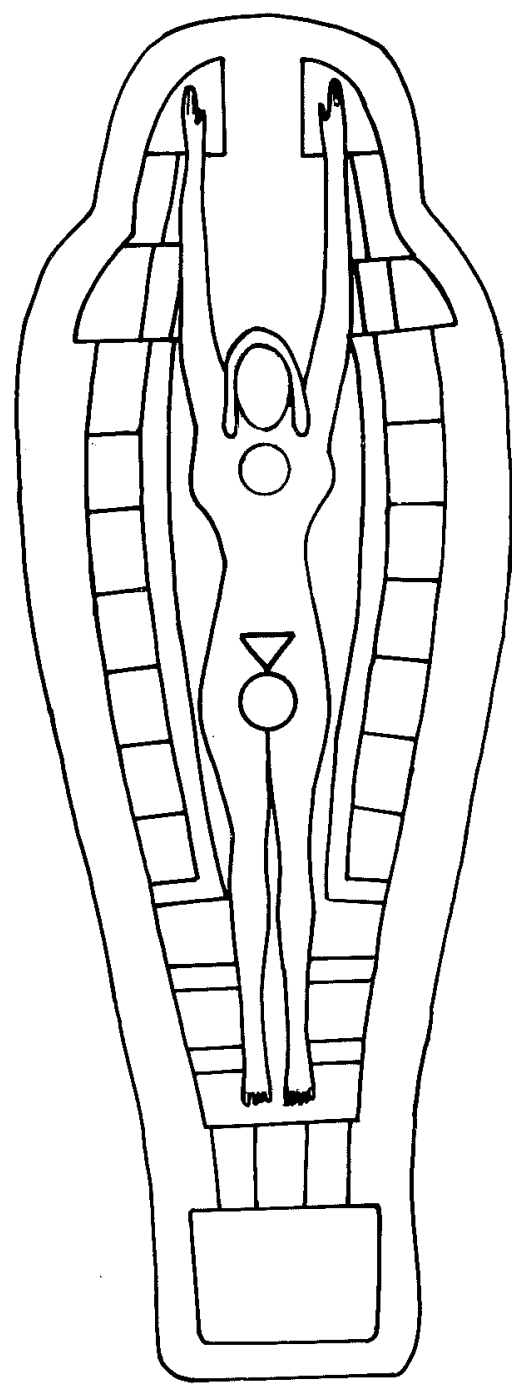

Fig.1

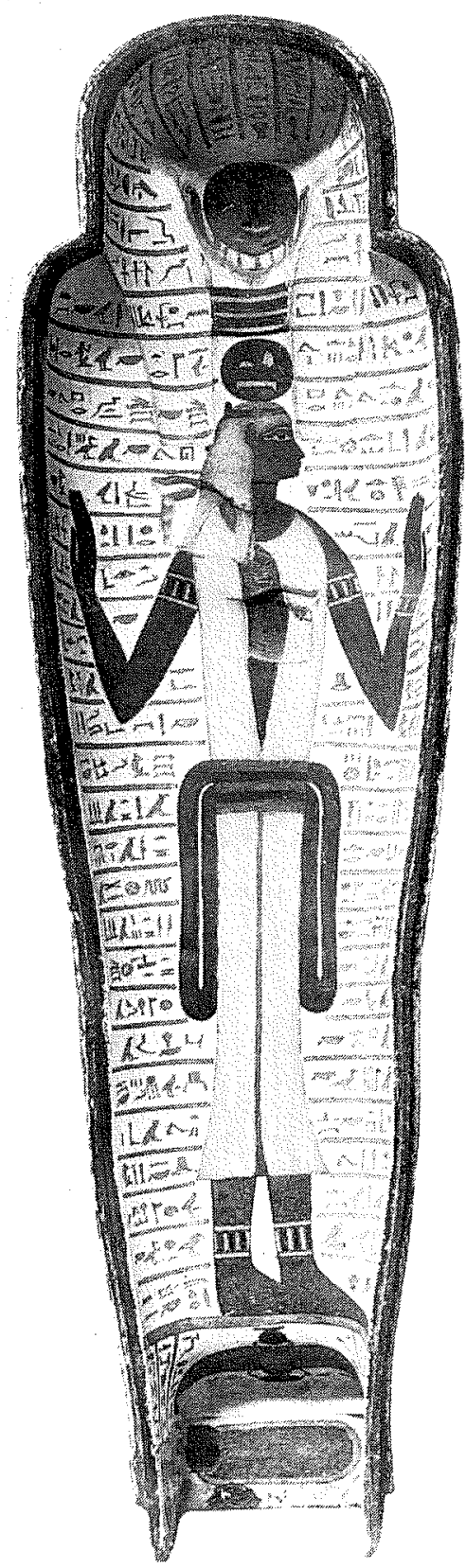

Fig. 2

Taf. 2. Nut an der Sargdecke.

Fig. 1. Summarische Abzeichnung aus dem Leidener Sarg M 13. Cf. Schmidt 218 Nr, 1238. (Der Leib ist eigentlich ganz schwarz und von Sternen bedeckt.)

Fig. 2. Aus dem Kairoer Sarg Nr. 41.042. Cf. Gauthier 2, Taf. 2; cf. 1, $13 \mathrm{ff}$. 




Fig.1

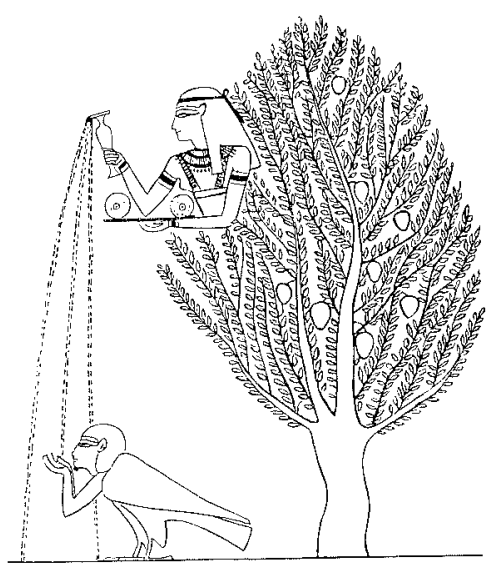

Fig. 2

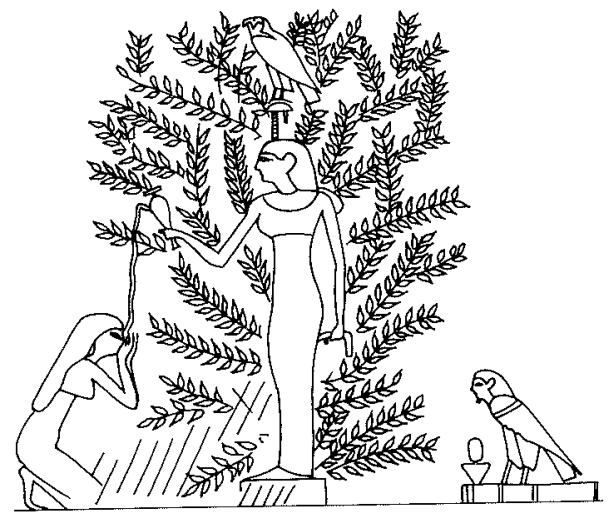

Fig. 3

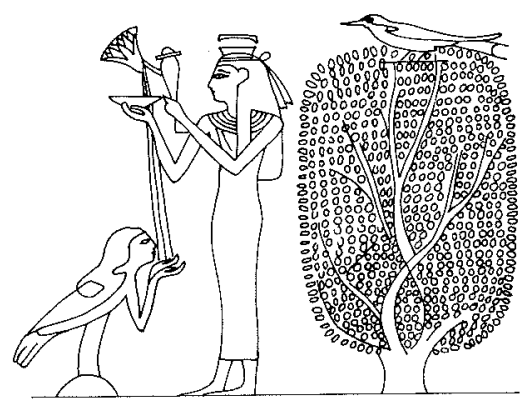

Fig. 4

Taf. 3. Nut - Baumgöttin.

Fig. 1. Relief aus Begerauieh, Nubien (ptolemäisch). Cf. Lepsius 36.

Fig. 2. Aus einem thebanischen Grab. Cf. Schmidt Titelvignette.

Fig. 3. Aus einer Mumienkiste in Marseille. Cf. Schmidt 153 Nr. 805.

Fig. 4. Aus einem Kairoer Sarg. Cf. Schmidt 153 Nr. 803. Cf. auch Englund 1974, 51 Fig. 7. 


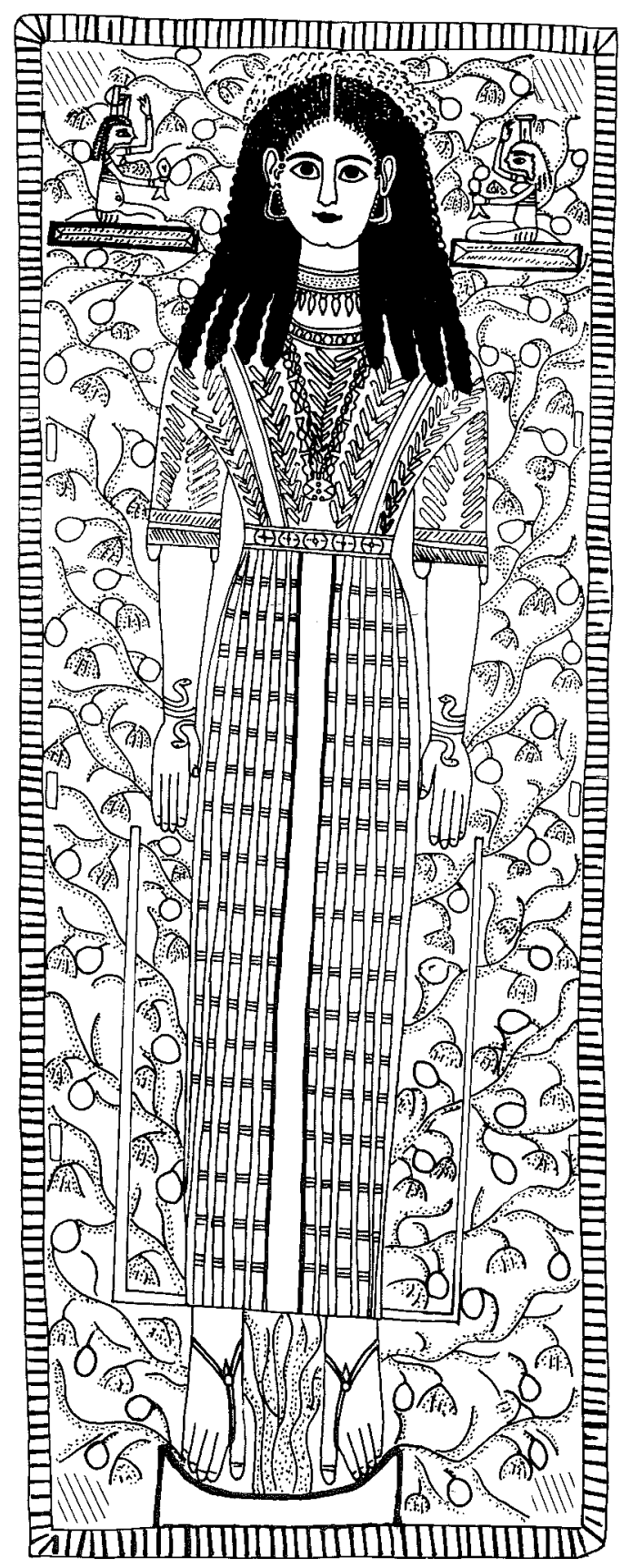

Taf. 4. Sargboden aus dem British Museum Nr. 6705 (römisch). Ich verdanke der Museumsverwaltung ein ausgezeichnetes Photo, nach dem diese Abzeichnung gemacht ist. Cf. auch Grimm Taf. 138,3

Bei der Herstellung der Abbildungen (Abzeichnungen usw.) ist mir Frau Britta Eriksson, erste Zeichnerin der Institution der Ägyptologie der Uppsala Universität behilflich gewesen. Für ihre ausgezeichnete Arbeit will ich ihr auch hier meinen besonderen Dank sagen. 
platten von Achmim der Fall. Dieses Verhältnis aktualisiert ein ikonographisches Problem, und zwar den augenfälligen Variantenreichtum in den Darstellungen des Motivs Göttin-Baum. M. W. ist dieses ikonographische Thema in dieser Hinsicht einmalig in der ganzen ägyptischen Darstellungswelt, die bekanntlich von einer gewissen Stereotypie geprägt ist, da der Ägypter gern an einer idealen Darstellungsart, die aus einem gewählten Sehwinkel bestimmt ist, festhält. Deshalb wären eine gründliche Inventierung dieser Szenen und eine detaillierte ikonographisch vergleichende Analyse dieser Dokumentation wertvoll. Hier müssen wir uns damit begnügen, nur einige Feststellungen im Anschluss an die wenigen gegebenen Abbildungen (Taf. 1 Fig. 2; Taf. 3 Fig. 1-4) zu machen und ausserdem nur die wichtigsten anderen Varianten anzudeuten.

Ziemlich ungewöhnlich ist die ganz lose Verbindung, in der Göttin und Baum nebeneinander dargestellt werden, ohne dass eine Beziehung zwischen ihnen angedeutet wird (Taf. 3 Fig. 4$)^{35}$. Eine maximal dendromorphe Ausgestaltung zeigt Figur 1 (Taf. 3) auf, wo zwei Wasserstrahlen ohne jede Andeutung einer Hand oder eines Armes aus dem Baum hervorströmen. Diese Darstellung scheint jedoch ziemlich unik und kann als missverstandene Ausgestaltung ausfallen, denn sie gehört einer entfernten äthiopischen Gegend und einer späten Epoche an. Andererseits kann die Verbindung der anthropomorph gestalteten Göttin mit dem Baum minimal sein, indem sie nur ein Baumzeichen auf dem Kopfe trägt, um ihre Baumnatur vage anzudeuten ${ }^{36}$. Zwischen diesen beiden Polen, dem ganz dendromorphen und dem ganz anthropomorphen, gibt es eine lange Reihe von Zwischenformen. So können nur Hände und vereinzelt (nur wenn Isis statt Nut auftritt) auch Brüste ${ }^{37}$, mit ihren Gaben aus dem Baum hervortreten (Taf. 1 Fig. 2, links), oder aber es wächst ein Göttinnenkopf aus dem Wipfel des Baumes hervor. In einigen Fällen ist etwa die Hälfte der Göttinnengestalt im Baum dargestellt (Taf. 3 Fig. 2), in anderen sieht man fast die ganze Gestalt oben im Zweigwerk stehen. Auch die anthropomorphe Ausgestaltung kann vorherrschend sein, so dass das Zweigwerk nur die Konturen der menschlichen Gestalt hervorhebt, bisweilen ganz lose mit dieser Gestalt verbunden (Taf. 3 Fig. 3). Manchmal ist der Baum zwar ziemlich füllig dargestellt, hat aber keinen anderen Stamm als die Beine der Göttin (Taf. 1 Fig. 2 rechts). Ein Durchgehen der gesammelten Dokumentation lässt fragen, ob dieser erstaunliche Variantenreichtum vielleicht

${ }^{35}$ Eine solche lose Gruppierung ist überhaupt im Alten $\ddot{A g y p t e n}$ selten zu belegen (cf. Bergman 1974, $95 \mathrm{ff}$.). Interessant ist die Darstellung Piankoff, Nr. 9, wo eine Beziehung dadurch etabliert wird, dass sowohl die Göttin als auch der Baum den Namen Nut trägt.

${ }^{36}$ Nach Buhl, 92, findet sich die früheste Darstellung dieser Art im Grab des Nakht.
Cf. auch das Grab des Userhet (Hinweis ib. Anm. 33) und das thebanische Grab Nr. 216 (Hinweis Schäfer 24 Abb. 6).

${ }^{37} \mathrm{Cf}$. das bekannte Graffito im Grab des Thutmosis III. (z. B. Mekhitarian, 38, abgebildet). Für diese Sonderdarstellung dürfte auch der Umstand, dass die Mutter des Pharao tatsächlich Isis hiess, von Belang gewesen sein. 
damit zusammenhängen könnte, dass die innere Triebkraft des Motivs gerade das Wachstum durch Ernährung ist.

Nebenbei soll ausserdem festgestellt werden, dass man keine einfachen Entwicklungslinien aufzeigen kann, die etwa von einer frühen fetischartigen dendromorphen Gestalt bis zu einer späteren, ,, höher entwickelten" anthropomorphen Darstellungsart führen. Bezeichnend ist, dass auf den ptolemäischen Opferplatten aus Achmim fast durchgehend zwei Baumszenen vorliegen, von denen die linke beinahe ganz dendromorph, die rechte aber hautpsächlich anthropomorph ist (Taf. 1 Fig. 2). Möglicherweise liegen hier verschiedene unter- und oberägyptische Sondertraditionen der Darstellungsweise vor.

\section{Verbindung der beiden Motive}

Bisher haben wir bei unserer Behandlung der beiden mit Nut verbundenen Komplexe der Lebensgebung jedes Sondermotiv für sich dargelegt und dessen Kontext und Funktion festgestellt. Hier soll nun die Frage gestellt werden, wie die beiden Vorstellungssphären sich zueinander verhalten. Die das Leben gebende Göttin ist ja dieselbe, es handelt sich um dasselbe Leben, das im funerären Kontext demselben Toten zugute kommt. Deshalb könnte man ja erwarten, dass enge Verbindungen der beiden Motive geläufig zu belegen sind. Dies ist aber nicht der Fall. Einige Begegnungspunkte können jedoch angegeben werden. Auf einigen Särgen heisst es, dass Nut dem Toten Speise und Trank gibt in einem Kontext, der eher zur Nut-Gebärerin-Sphäre gehört ${ }^{38}$. An der Decke des thebanischen Grabes Nr. 216 finden wir die Ernährerin Nut - sie trägt die Sykomore auf dem Kopfe und giesst Wasser aus zwei Libationsgefässen aus $^{39}$ - an einer Stelle, wo eigentlich die Himmelsgöttin als Gebärerin ihren rechten Platz hätte. Bisweilen finden sich auf Särgen Darstellungen, die je einer der beiden Vorstellungssphären angehören, in der Nähe von einander ${ }^{40}$. Oft trägt auch die Baumgöttin Nut die Bezeichnung ,,diejenige, die die Götter gebiert". Es sei ausserdem daran erinnert, dass die Wasserlibation der Nut mit ihrem Wesen als Himmelsozean verbunden werden könnte, einer Vorstellung, von der Sonnenschiffe und Sternenbarken, die auf dem Leib der Göttin einherfahren, zeugen. Auf einem Pariser Eimer ${ }^{41}$ finden wir Nut/ die Sykomore frontal dargestellt, was eher, wie oben betont wurde, die Gebärerin Nut kennzeichnet.

Ein Sargboden des Pollios Soter-Grabfundes aus einer thebanischen Nekropole, jetzt im British Museum ausgestellt (N:r 6705), ist für uns von besonderem Interesse, wie Taf. 4 zeigt. Den Hintergrund der Hauptfigur

${ }^{38}$ Cf. Rusch 52.

${ }^{39}$ Cf. Schäfer 24 Abb. 6 (cf. auch die hiermit zusammenhörende Abb. 5).

${ }^{40} \mathrm{Z}$.B. die Szenen 2 und 4 , rechts, auf dem
Sarg des Chonsumes in Uppsala (cf. Englund 1974,50 ff.).

${ }^{41}$ Abgebildet Parrot 115, 119. 
bildet ein aus dem Horizont hervorwachsender Baum mit roten Früchten. Man hat diese Baumdekoration des Sargbodens, die sich in Ägypten nicht früher belegen lässt, als Einfluss des griechisch-römischen Kulturkreises erklären wollen ${ }^{42}$. Dass die Darstellung solche Spuren aufzeigt (z. B. Schlangenarmband, Wiedergabe des Haares und der Gewandung), ist klar. Was den Baum anbelangt, bin ich aber der Meinung, dass wir es hier mit der oben behandelten Vorstellung von Nut als Baumgöttin zu tun haben. Ich will in diesem Zusammenhang daran erinnern, dass Osiris beim Choiakfest in der Spätzeit auf einem Bett von Sykomorenzweigen ruhen sollte. Dieses rituelle Verfahren lässt sich trefflich mit dem Umstand vereinen, dass die betreffende Darstellung sich gerade auf dem Boden des Sarges befindet. Die Hauptfigur trägt keinen Namen, aber eine Reihe von Särgen aus der gleichen Zeit stellen sicher, dass es hier um Nut geht ${ }^{43}$. Augenfällig ist jedoch der Tatbestand, dass es sich hier nur um Frauensärge handelt. Deshalb ist es wahrscheinlich, dass die Darstellung eine durch die funerären Zeremonien mit der Göttin Nut identifizierte Frau abbildet ${ }^{44}$. Dieses Verhältnis könnten auch die oben angegebenen griechisch-römischen Züge unterstreichen. Wenn diese Deutung richtig ist, sollten die alten ägyptischen Nut-Züge und die modernen Kennzeichen der betreffenden Frau zusammen diese neue Identität sicherstellen. Dieses Nut-Werden dürfte eine Parallelerscheinung sein zum vereinzelt belegten Brauch, in dieser Zeit eine mumifizierte Frau als Hathor NN - nicht als Osiris NN - zu bezeichnen. Wenn man die verbreitete, tiefgreifende Symbolik bedenkt, die sich um die Göttin Nut als Lebensgeberin im alten Ägypten entwickelte und durch Darstellungen und Kultbräuche immer mehr befestigt wurde, ist es leicht zu verstehen, warum die Frauen wünschten, gerade mit dieser Göttin identifiziert zu werden ${ }^{45}$.

Die Durchschlagskraft dieser Vorstellungwelt, in der Nut nicht nur als die Lebensgeberin par exellence sondern sogar als das Leben selbst hervortrat, lässt sich vielleicht noch weiter verfolgen. M. E. kann man diese Symbolik als eine Art Anch-Mystik hinter der merkwürdigen Korrespondanz zwischen der Gestalt der Toten und dem Anch-Zeichen auf der koptischen Grabstele der Rhodia ${ }^{46}$ spüren. Um dieses aufzuzeigen, ist aber eine Sonderstudie nötig.

${ }^{42}$ Cf. Grimm, 118 Anm. 156, gibt auch einige Paralleldarstellungen an, von denen der Sargboden im Louvre (Taf. 139,1) die grösste Verwandtschaft mit dem unsrigen aufzeigt.

${ }^{43}$ Cf. Grimm Taf. $138 \mathrm{f}$.

${ }^{44}$ Cf. Parlasca 161, 166.

${ }^{45}$ In einem späten Louvre-Papyrus (Nr. 3148), nach dem Nut als Mutter und Sarg spricht (cf. Schott $81 \mathrm{ff}$.), sagt sie: ,Deine Mutter sagt: Deine Mutter hat dich $10 \mathrm{Mo}-$ nate getragen, sie hat dich drei Jahre genährt.
Ich trage dich eine unbestimmte Zeit. Ich werde dich nie gebären." Die letzte seltsame Aussage könnte vielleicht so erklärt werden, dass die Tote nun mit Nut für ewig vereinigt ist und so mit ihr praktisch identifiziert.

${ }^{46}$ Cramer, 37, (cf. auch Tafel 16 Abb. 33) liefert nur eine kurze Beschreibung dieser Stele, ohne die Verwendung des AnchZeichens in den von mir angedeuteten weiteren Symbolkontext einzustellen. 


\section{Literaturverzeichnis}

CT Buck, A. de, The Egyptian Coffin Texts I-VII. Chicago 1935-1961.

FG From the Gustavianum collections in Uppsala, ed. S. Brunnsåker, H.-A. Nordström. Uppsala 1974.

Pyr. Sethe, K., Die altägyptischen Pyramidentexte 1-4. Leipzig 1908-1922.

RÄRG Bonnet, H., Reallexikon der ägyptischen Religion. Berlin 1952.

Urk. Sethe, K. (uam.), Urkunden des ägyptischen Altertums. Leipzig $1903 \mathrm{ff}$.

ZÄS Zeitschrift für ägyptische Sprache und Altertumskunde.

Baines, J., 1975, 'Ankh-Sign, Belt and Penis Sheath. Studien zur altägyptischen Kultur 3.

Bergman, J., 1970, Isis-Seele und Osiris-Ei. Uppsala.

- 1970a, Mystische Anklänge in der altägyptischen Vorstellungen von Gott und Welt. Mysticism, ed. S. Hartman, C.-M. Edsman. Scripta Instituti Donneriani Aboensis 5. Uppsala.

- 1974, Isis auf der Sau. $F G$.

- 1975, Quelques réflexions sur $n f r-n f r . t-n f r w$. Congrès international des Orientalistes. Egyptologie 1. Paris.

Brunner, H., 1964, Die Geburt des Gottkönigs. Wiesbaden.

Buck, A. de, 1947, Plaats en betekenis van Sjoe in de egyptische theologie, Amsterdam.

Buhl, M.-L., 1947, The goddesses of the Egyptian tree cult. Journal of Near Eastern studies 6.

Cramer, M., 1955, Das altägyptische Lebenszeichen im christlichen (koptischen) Ägypten. Wiesbaden.

Doresse, J., 1960, Des hiéroglyphes à la croix. Istambul.

Englund, G., 1974, Propos sur l'iconographie d'un sarcophage de la $21^{\mathrm{e}}$ dynastie. $F G$.

- 1978, Ach - une notion religieuse dans l'Egypte pharaonique. Uppsala.

Fischer, H. G., 1972, Some emblematic uses of hieroglyphs with particular reference to an archaic ritual vessel. The Metropolitan Museum journal 5.

- 1973, An eleventh dynasty couple holding the sign of life. $Z \ddot{A} S 100$.

Frankfort, H., 1933, The cenotaph of Seti I at Abydos. London.

Gauthier, H., 1913, Cercueils anthropoïdes des Prêtres de Montou 1-2. Kairo.

Grapow, H., 1935, Die Himmelsgöttin Nut als Mutterschwein. ZÄS 71.

Grimm, G., 1974, Die römischen Mumienmasken aus Ägypten. Wiesbaden.

Hayes, W., 1953, The sceptre of Egypt 1 . New York.

Helck, W., Otto, E., 1972, Lexikon der Ägyptologie 1. Wiesbaden.

Hornung, E., 1963 f, Das Amduat 1-3. Wiesbaden.

Jéquier, G., 1894, Le Livre de ce qu'il y a dans l'Hadès. Paris.

- 1922, Matériaux pour servir à l'établissement d'un dictionnaire archéologique égyptienne. Bulletin de l'Institut français d'archéologie Orientale 19.

Junker, H., 1913, Das Götterdekret über das Abaton. Wien.

- 1954, ,,Der Lebendige“ als Gottesbeiname im Alten Reich. Anzeiger der österreichischen Akademie der Wissenschaften 12.

Kamal, A. B., 1909, Tables d'offrandes 1-2. Kairo.

Kaplony, P., 1963, Die Inschriften der ägyptischen Frühzeit 3. Wiesbaden.

Lepsius, R., $1849 \mathrm{ff}$, Denkmäler aus Ägypten und Äthiopien. Atlas 5. Berlin.

Mariette, A., 1872, Monuments divers. Paris.

Mekhitarian, A., 1954, Ägyptische Malerei. Genf.

Moftah, R., 1965, Die uralte Sykomore und andere Erscheinungen der Hathor. $Z \ddot{A} S 40 \mathrm{ff}$. 
Parlasca, K., 1966, Mumienporträts und verwandte Denkmäler. Wiesbaden.

Parrot, A., 1937, Le "Refrigerium » dans l'au-delà. Paris.

Piankoff, A., Rambova, Natacha, 1957, Mythological Papyri. New York.

Rusch, A., 1922, Die Entwicklung der Himmelsgöttin Nut zu einer Totengottheit. Leipzig.

Schäfer, H., 1935, Altägyptische Bilder der auf- und untergehenden Sonne. ZÄS 71.

Schmidt, W., 1919, Levende og Døde i det gamle Aegypten. Kopenhagen.

Schott, S., 1965, Nut spricht als Mutter und Sarg. Revue d'Egyptologie 17.

Wallert, I., 1962, Die Palmen im alten Ägypten. Berlin.

Westendorf, W., 1966, Beiträge aus und zu den medizinischen Texten. $Z \ddot{A} S 92$.

Winter, E., 1968, Untersuchungen zu den ägyptischen Tempelreliefs der griechischrömischen Zeit. Wien. 